\title{
Ventilatory Response to Hypoxia during Endotoxemia in Young Rats: Role of Nitric Oxide
}

\author{
JOHN LADINO, EDUARDO BANCALARI, AND CLEIDE SUGUIHARA
} Department of Pediatrics, Division of Neonatology, Neonatal Developmental Biology Laboratory, Batchelor Children's Research Institute,
University of Miami Miller School of Medicine, Miami, Florida 33101

\begin{abstract}
Administration of Escherichia coli endotoxin attenuates the ventilatory response to hypoxia (VRH) in newborn piglets, but the mechanisms responsible for this depression are not clearly understood. Nitric oxide (NO) production increases during sepsis and elevated NO levels can inhibit carotid body function. The role of endothelial $\mathrm{NO}$ on the VRH during endotoxemia was evaluated in 26 young rats. Minute ventilation $\left(\mathrm{V}_{\mathrm{E}}\right)$ and oxygen consumption $\left(\mathrm{VO}_{2}\right)$ were measured in room air (RA) and during $30 \mathrm{~min}$ of hypoxia $(10 \%$ $\mathrm{O}_{2}$ ) before and after $E$. coli endotoxin administration. During endotoxemia, animals received placebo (PL, $n=8$ ); a nonselective nitric oxide synthase (NOS) inhibitor ( $N^{G}$-nitro-L-arginine methyl ester, L-NAME, $n=9$ ), or a neuronal NOS (nNOS) inhibitor (7nitroindazole, 7-NI, $n=9$ ). During endotoxemia, a larger increase in $\mathrm{V}_{\mathrm{E}}$ was observed only during the first min of hypoxia in the L-NAME group when compared with PL or 7-NI $(p<0.001)$. VRH was similar in the PL and 7-NI groups. A larger decrease in $\mathrm{VO}_{2}$ at $30 \mathrm{~min}$ of hypoxia was observed in L-NAME and 7-NI groups when compared with PL $(p<0.03)$. These data demonstrate that the attenuation of the early VRH during endotoxemia is in part mediated by an inhibitory effect of endothelial NO on the respiratory control mechanisms. (Pediatr Res 62: 134-138, 2007)
\end{abstract}

$\mathrm{C}$ hanges in breathing pattern and apnea episodes are frequently observed in newborns and infants with sepsis (1). In adult humans, endotoxin administration results in changes in the basal respiratory pattern and dyspnea (2). We previously demonstrated that endotoxin infusion in newborn piglets produced attenuation of the VRH (3). In a pilot study, we also observed similar respiratory behavior in 4- to 6-wkold rats. The interaction of hypoxia, infection, and cytokine abnormalities has been suggested as a possible mechanism of sudden infant death syndrome (SIDS) (4).

The mechanisms that explain the changes in breathing pattern during sepsis are not clearly understood. Respiratory muscle fatigue or alterations in lung mechanics and metabolic rate have been associated with the respiratory changes observed during Gram-positive and negative septicemia in piglets $(3,5,6)$. Particularly, the attenuation in the VRH observed after endotoxin administration to unanesthetized newborn piglets was associated with a significant decrease in $\mathrm{VO}_{2}$ (3). Sepsis can induce the release of inflammatory mediators such

Received November 8, 2006; accepted March 26, 2007

Correspondence: Cleide Suguihara, M.D., University of Miami Miller School of Medicine, Department of Pediatrics (R-131), P.O. Box: 016960, Miami, FL 33101; e-mail: csuguihara@med.miami.edu

Supported by the University of Miami: Project New Born. as cytokines; prostaglandins, and NO $(7,8)$, which may be responsible for the alterations of the breathing pattern observed during endotoxemia and also may be an important component of many deaths attributed to SIDS (4).

It is known that NO is an active participant in the pathophysiology of endotoxemia (9). NO is synthesized from Larginine by three different isoenzymes of NOS: neuronal NOS (nNOS), inducible NOS (iNOS) and endothelial NOS (eNOS) (10). Increased eNOS activation and endothelial NO release have been observed shortly after endotoxin administration $(11,12)$. Elevated NO expression has been found to be associated with organ damage described in autopsy reports of infants who died of SIDS. NOS mRNA up-regulation has been observed after endotoxin administration in animal models for the study of SIDS $(13,14)$.

In vitro and in vivo studies indicated that $\mathrm{NO}$ has inhibitory properties at the carotid body and that eNOS is primarily responsible for this effect $(15,16)$. Administration of $\mathrm{NO}$ donors to the carotid body reduces its chemosensory response to a decrease in oxygen concentration (16). Studies in rats have also shown that NO could depress the early VRH (15). It has been proposed that during hypoxia, a decreased synthesis of an inhibitory chemical messenger such as NO may contribute to the augmentation of sensory discharge observed in animals exposed to low oxygen concentrations (17).

Based on these observations, we hypothesized that the attenuation of the VRH observed after Escherichia coli endotoxin administration to unanesthetized young rats may be modulated by an increase in NOS, specifically eNOS. Furthermore, this attenuated VRH may be associated with changes in $\mathrm{VO}_{2}$. The objective of this study was to evaluate the effect of eNOS inhibition on the ventilatory and metabolic responses to hypoxia during endotoxemia.

\section{MATERIALS AND METHODS}

Animals. Twenty-five female Wistar rats, ages 4-5 wk, were obtained from Charles-River Laboratories (Wilmington, MA). The rats were fed rat chow and water ad libitum, kept on a light-dark cycle of 12:12 h, and studied at 5-6 wk of age. The procedures used in the care and handling of the animals were in accordance with the guidelines of the National Institutes of Health.

Abbreviations: eNOS, endothelial NOS; L-NAME, $N^{G}$-nitro-L-arginine methyl ester; nNOS, neuronal NOS; NO, nitric oxide; NOS, nitric oxide synthase; PL, placebo; RA, room air; RR, respiratory rate; 7-NI, 7-nitroindazole; $\mathbf{T b}$, body temperature; $\mathbf{V}_{\mathbf{E}}$, minute ventilation; $\mathbf{V O}_{\mathbf{2}}$, oxygen consumption; VRH, ventilatory response to hypoxia; $\mathbf{V}_{\mathbf{T}}$, tidal volume 
The study protocol was reviewed and approved by the Animal Care and Use Committee of the Miller School of Medicine, University of Miami.

Ventilatory measurements. Ventilation was measured by using whole body flow plethysmography for unrestrained, unanesthetized small rodents as previously described (model PLY3213, Buxco Electronics, Sharon, CT) $(18,19)$. Briefly, pressure differences across two screen mesh pneumotachographs located on top of the plethysmograph were measured using a flow transducer. With appropriate software, the recorded flow signal was then integrated on a breath-by-breath basis to obtain a relative $V_{T}, R R$, and $V_{E}$ (Biosystem XA Pulmonary Analyzer, Buxco Electronics) $(18,19)$.

Behavioral states were continuously scored using standard behavioral criteria (20). During circadian cycle, the nonrestrained and undisturbed rats spend most of the daytime in a sleep state, while at night they are awake and active. The rats were deemed to be either awake (eyes open with gross body movements, such as crawling or grooming), in quiet sleep (eyes closed, recumbent with limbs adducted, regular and stable respiratory rhythm with minimal or no sighs or periodic breathing and absence of movements except for intermittent brief startles) or in active sleep (recumbent, eyes closed, irregular respiratory pattern, frequent twitches of the whiskers, ears, and extremities). Eye movements were not used since it was difficult to see these through the plethysmograph (20). These observations were made to ensure that the measurements were not obtained during active sleep, which can modify the ventilatory response to hypoxia (19). Nevertheless, active sleep in rats accounts for only about $15 \%$ of total daytime sleep and this component of sleep decreases during hypoxia (21).

Metabolic measurements. $\mathrm{VO}_{2}$ was measured by the open-flow method using the plethysmograph as a metabolic box (22). The gas atmosphere within the plethysmograph was maintained with a bias flow of RA or at a fractional concentration of inspired $\mathrm{O}_{2}\left(\mathrm{FiO}_{2}\right)$ of 0.1 . A constant flow rate $\left(\mathrm{V}_{\mathrm{s}}\right)$ was maintained during the experiment and it was measured before and after each run by a Matheson linear mass flowmeter $(0-20 \mathrm{~L} / \mathrm{min}$, model 8100 , Matheson Gas Products, Secaucus, NJ). The excurrent flow was passed through a drying column (Drierite, W.A. Hammond Drierite Co. Ltd., Xenia, OH) before entering a previously calibrated $\mathrm{O}_{2}$ analyzer (Ametek S-3A/I, Pittsburgh, PA) to measure the fractional concentration of expired $\mathrm{O}_{2}\left(\mathrm{FeO}_{2}\right) . \mathrm{VO}_{2}$ was calculated by the following formula: $\mathrm{VO}_{2}=\mathrm{V}_{\mathrm{s}} \times\left(\mathrm{FiO}_{2}-\mathrm{FeO}_{2}\right)$ at standard temperature and ambient pressure. The metabolic measurements were digitized by AT-CODAS (Dataq Instruments, Akron, $\mathrm{OH}$ ) at a frequency of $100 \mathrm{~Hz}$ and recorded into a microprocessor for later analysis. $\mathrm{Vo}_{2}$ values are reported at $30 \mathrm{~min}$ of hypoxia, to allow enough time for complete equilibrium of the gases inside the box.

Experimental protocol. To ensure reproducibility of the findings, one day before the experiment the animals were placed in the plethysmograph for intermittent periods of $1 \mathrm{~h}$. Testing was performed between 0800 and $1500 \mathrm{~h}$ to limit circadian effects. On the day of the experiment, the animals were placed in the plethysmograph and allowed to acclimatize for $60 \mathrm{~min}$. Ambient temperature inside the box was maintained between 23 and $25^{\circ} \mathrm{C}$, values in the range of thermoneutrality for Wistar rats. Tb was measured by inserting a rectal thermistor probe (YSI Inc., Yellow Springs, $\mathrm{OH}$ ) and values were also recorded throughout the study protocol. After randomization to the experimental groups, animals received a dose of placebo (PL) $(1 \mathrm{~mL}$ normal saline intraperitoneal) and baseline measurements of $\mathrm{V}_{\mathrm{E}}, \mathrm{RR}, \mathrm{V}_{\mathrm{T}}$, and $\mathrm{VO}_{2}$ were recorded in RA for $30 \mathrm{~min}$ (Fig. 1). Measurements were repeated again during 30 min of hypoxia $\left(\mathrm{FiO}_{2}=0.10\right)$ that was induced by rapidly flushing the box with a precalibrated $\mathrm{O}_{2}-\mathrm{N}_{2}$ gas mixture $(15 \mathrm{~L} / \mathrm{min}$ in $15 \mathrm{~s})$. The animals were allowed to rest for $120 \mathrm{~min}$ by being returned to their cages and being permitted to move, drink and feed ad libitum. Afterward, they received an intraperitoneal injection of E. coli O55:B5 endotoxin (Sigma Chemical Co., St. Louis, MO) at a dose of $25 \mu \mathrm{g} / \mathrm{kg}$ in $1 \mathrm{~mL}$ saline. The selected dose of endotoxin and the time frame for the study were based on pilot studies, which



Figure 1. Experimental protocol. $\mathrm{M} 1-\mathrm{M} 4: \mathrm{V}_{\mathrm{E}}, \mathrm{V}_{\mathrm{T}}, \mathrm{RR}, \mathrm{V}_{2}$ measurements. M1 and M3: baseline. demonstrated no significant changes in basal $\mathrm{V}_{\mathrm{E}}$ with this dose of endotoxin In addition, this dose of endotoxin was below the threshold that causes changes in blood pressure or endotoxic shock in the rat (23). After $150 \mathrm{~min}$ endotoxin administration, each animal in its respective experimental group received either an intraperitoneal injection of PL or $100 \mathrm{mg} / \mathrm{kg}$ of L-NAME, a nonspecific NOS inhibitor or $25 \mathrm{mg} / \mathrm{kg}$ of 7-NI, a selective nNOS inhibitor. The selected doses of NOS inhibitors have been previously validated in the rat $(24,25)$. Immediately after PL, L-NAME, or 7-NI administration, the animals were returned to the plethysmograph and baseline measurements were recorded again in room air for $30 \mathrm{~min}$. Finally, measurements were repeated during 30 min of hypoxia.

Data analysis. Ventilation and $\mathrm{VO}_{2}$ values obtained at room air immediately before hypoxia challenge were considered as baseline values. The changes in ventilation and $\mathrm{VO}_{2}$ during hypoxia were expressed as percent change from baseline values. Paired $t$ test was used for comparison of measurements during hypoxia pre- and post-endotoxin administration in the PL group. Bonferroni correction was used to correct for the effect of multiple comparisons. Comparison of measurements post-endotoxin administration between PL versus L-NAME or 7-NI, and L-NAME versus 7-NI groups was performed by two-sample $t$ test. Data are reported as mean $\pm \mathrm{SD}$ and a $p$ value $<0.05$ was considered statistically significant.

\section{RESULTS}

Nine rats (age $5.3 \pm 0.5 \mathrm{wk}$; weight $143 \pm 17 \mathrm{~g}$ ) received L-NAME, 9 rats (age $5.1 \pm 0.4 \mathrm{wk}$; weight $136 \pm 16 \mathrm{~g}$ ) received 7-NI and 8 rats (age $5.5 \pm 0.5 \mathrm{wk}$; weight $143 \pm$ $21 \mathrm{~g})$ received PL. There was no significant difference in age and weight among groups.

Baseline values for $\mathrm{V}_{\mathrm{E}}$ did not differ among the studied groups before (PL: $105 \pm 19 \mathrm{~mL} / \mathrm{min} / 100 \mathrm{~g}$; L-NAME: $117 \pm$ $9 \mathrm{~mL} / \mathrm{min} / 100 \mathrm{~g}$; 7-NI: $107 \pm 9 \mathrm{~mL} / \mathrm{min} / 100 \mathrm{~g}$ ) and after (PL: $120 \pm 19 \mathrm{~mL} / \mathrm{min} / 100 \mathrm{~g}$; L-NAME: $119 \pm 12 \mathrm{~mL} / \mathrm{min} / 100 \mathrm{~g}$; 7-NI: $116 \pm 17 \mathrm{~mL} / \mathrm{min} / 100 \mathrm{~g}$ ) endotoxin administration. The VRH before endotoxin administration was similar among the study groups (Fig. 2). As expected, the VRH after endotoxin administration was significantly attenuated during the first $(p<0.001)$ and subsequent $30 \mathrm{~min}$ in the PL group $(p<0.03)$ (Fig. 3).

After endotoxin administration, a significantly larger increase in $\mathrm{V}_{\mathrm{E}}$ was observed during the first min of hypoxia following NOS inhibition by L-NAME, when compared with PL $(p<0.03)$ (Fig. 3). This change was due to increases in both $\mathrm{V}_{\mathrm{T}}(40 \pm 29 \%$ versus $29 \pm 13 \%$, L-NAME versus $\mathrm{PL})$ and RR $(36 \pm 47$ versus $15 \pm 22 \%$, L-NAME versus $\mathrm{PL})$ during the first min of hypoxia. A similar attenuation in the

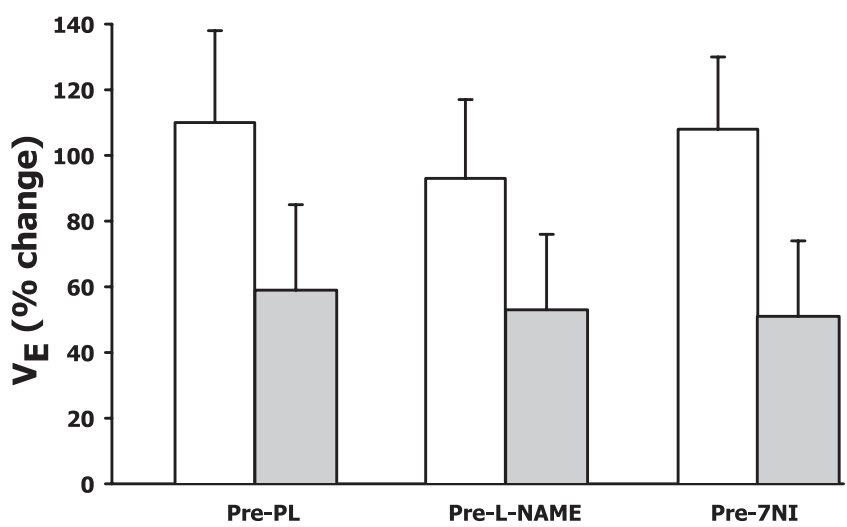

Figure 2. Changes in $V_{E}$ during hypoxia before endotoxin and PL, L-NAME, or 7-NI administration. The ventilatory response to hypoxia before endotoxin administration was similar at $1 \mathrm{~min}$ (open bars) and $30 \mathrm{~min}$ (shaded bars) of hypoxia in all study groups. 


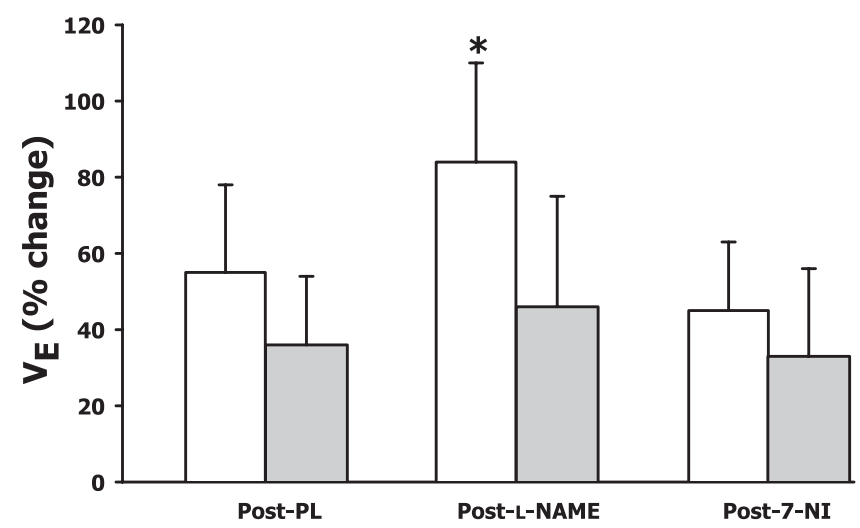

Figure 3. Changes in $\mathrm{V}_{\mathrm{E}}$ during hypoxia after endotoxin and PL, L-NAME, or 7-NI administration. The VRH was attenuated during the first min of hypoxia (open bars) after endotoxin administration in the PL and 7-NI groups. In contrast, the VRH during the first min of hypoxia was increased in the L-NAME group. The VRH did not differ significantly at $30 \mathrm{~min}$ of hypoxia (shaded bars) among groups. * $p<0.03$ : L-NAME $v s$ PL or 7-NI.

VRH was observed at 1 min of hypoxia in the PL and 7-NI groups after endotoxin administration (Fig. 3). Changes in $\mathrm{V}_{\mathrm{E}}$ at $30 \mathrm{~min}$ of hypoxia after endotoxin administration were not different among the three study groups (Fig. 3).

Baseline values for $\mathrm{VO}_{2}$ were not different among groups before (PL: $38 \pm 6 \mathrm{~mL} / \mathrm{min} / \mathrm{kg}$; L-NAME: $46 \pm 13 \mathrm{~mL} / \mathrm{min} / \mathrm{kg}$; 7-NI: $42 \pm 5 \mathrm{~mL} / \mathrm{min} / \mathrm{kg}$ ) and after (PL: $36 \pm 5 \mathrm{~mL} / \mathrm{min} / \mathrm{kg}$; L-NAME: $37 \pm 9 \mathrm{~mL} / \mathrm{min} / \mathrm{kg}$; 7-NI: $33 \pm 7 \mathrm{~mL} / \mathrm{min} / \mathrm{kg}$ ) endotoxin administration. The decrease in $\mathrm{VO}_{2}$ at $30 \mathrm{~min}$ of hypoxia before endotoxin administration was not significantly different among groups (PL: $-39 \pm 20 \%$, L-NAME: $-49 \pm$ 11\%, 7-NI: $-44 \pm 16 \%)$. After endotoxin administration during normoxia, $\mathrm{V}_{2}$ was not significantly different among groups. There was a decrease in $\mathrm{VO}_{2}$ at 30 min of hypoxia in the PL group $(n=7 ;-32 \pm 11 \%)$. However, a more pronounced and similar decrease in $\mathrm{VO}_{2}$ was observed in the L-NAME $(n=8 ;-46 \pm 25 \%)$ and 7-NI $(n=6 ;-45 \pm 16 \%)$ groups when compared with PL $(p<0.03)$ (Fig. 4).

Changes in $\mathrm{Tb}$ during hypoxia were similar among groups before endotoxin administration. After endotoxin was given, administration of the NOS inhibitors produced a significant decrease in Tb in RA (L-NAME: $37.5 \pm 0.3^{\circ} \mathrm{C}$, 7-NI: $37.2 \pm$

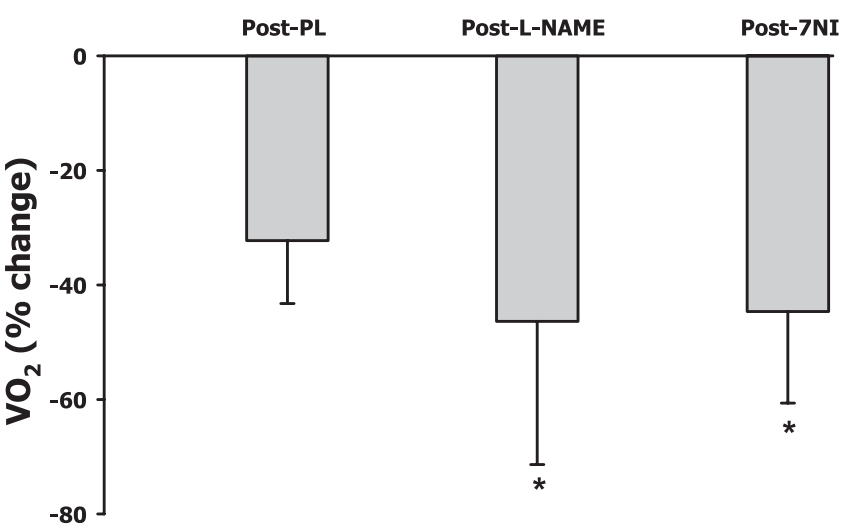

Figure 4. Changes in $\mathrm{VO}_{2}$ at $30 \mathrm{~min}$ of hypoxia after endotoxin and PL, L-NAME, or 7-NI administration. A greater decrease in $\mathrm{VO}_{2}$ during hypoxia after endotoxin administration was observed in L-NAME and 7-NI groups compared with PL. * $p<0.03$ : PL $v s$ L-NAME or 7-NI. $\left.0.6^{\circ} \mathrm{C}\right)$ when compared with PL $\left(38 \pm 0.5^{\circ} \mathrm{C}, p<0.05\right)$. However, after endotoxin administration, changes in Tb during hypoxia when compared with baseline after PL, L-NAME, or 7-NI administration were not different among groups.

\section{DISCUSSION}

The present study demonstrates a marked attenuation in the ventilatory response to hypoxia after $E$. coli endotoxin administration in unanesthetized, unrestrained young rats. This was observed throughout the hypoxic exposure, suggesting that $E$. coli endotoxin has both peripheral and central depressant effects on the respiratory control mechanisms. These data also suggest that the attenuation of the early VRH induced by endotoxin administration is in part mediated by an inhibitory effect of NO on the respiratory control mechanisms.

The attenuation in ventilation was more marked during the first min of hypoxia suggesting an inhibitory effect of endotoxemia on the peripheral chemoreceptors. This effect was reversed after administration of L-NAME, a nonspecific NOS inhibitor. This suggests that the early depression of the VRH after endotoxin administration is mediated by activation of the eNOS, because L-NAME attenuated the ventilatory depression whereas nNOS inhibition did not affect the response.

It is well known that NO plays a pathogenic role during sepsis and our results indicate that eNOS is involved in the early manifestations of endotoxemia. This is in agreement with different experimental models that have shown that endotoxin administration induces an early increase in eNOS and NO production $(8,11)$. At later stages, however, it has been demonstrated that eNOS is down-regulated whereas iNOS is up-regulated and is probably responsible for some of the cardiovascular effects attributable to the increased NO production during endotoxemia $(8,10)$. Because $\mathrm{NO}$ production is increased during endotoxemia, it can inhibit the peripheral chemoreceptor discharge and may mediate the attenuation in ventilation observed after endotoxin administration during the first min of hypoxia. These results are in agreement with previous observations showing that administration of $\mathrm{NO}$ donors to an in vitro carotid body preparation reduced its chemosensory response to a decrease in oxygen concentration but not to normoxia (16). If a decrease of the inhibitory properties of $\mathrm{NO}$ accounts for the initial increase in ventilation during hypoxia, the increase in NO production during Gramnegative infection may produce inhibition of carotid body activity during hypoxia with the consequent depression of the VRH. Nitric oxide, once released, can affect carotid body sensory activity by acting directly on the glomus cells or by changing blood flow at the level of the carotid body (17). Another possible pathway by which NO can exert its action on the carotid body is by an increase in the intracellular levels of cGMP with changes in the function of the calcium channels. This may lead to a further inhibition of the sensory discharge and a decreased excitatory neurotransmitter release (i.e. acetylcholine, adenosine nucleotides, and dopamine) $(17,26)$. Nitric oxide can also influence ventilation during hypoxia through a central mechanism. NO derived from central nNOS enhanced phrenic nerve output during hypoxia (27) and sys- 
temic administration of NOS inhibitors to rats decreased the late hypoxic ventilatory response (28). In the present study, neither L-NAME nor 7-NI administration produced significant changes in the late $(30 \mathrm{~min})$ ventilatory depression observed during hypoxia after endotoxin administration. This suggests that NO does not influence the late VRH after endotoxin administration.

The ventilatory depression during hypoxia observed after endotoxin could be associated with increased production of other mediators, such as platelet derived growth factor, IL-1 $\beta$ or prostaglandins at the level of the CNS. Their activation can depress respiration and change the breathing pattern during hypoxia (28-30). Their expression is also increased during endotoxemia $(7,30)$ and consequently could explain the observed late depression in the VRH after endotoxin administration. This possibility requires further investigation to be elucidated.

In a previous study in newborn piglets, we found that the attenuation of the hypoxic ventilatory response following endotoxin infusion was associated with a greater decrease in metabolic rate (3). However, in the present study in young rats, the changes in $\mathrm{VO}_{2}$ were similar before and after endotoxin in the PL group. These dissimilar findings may be related to differences in maturational stages or in animal species. Interspecies variations of $\mathrm{V}_{\mathrm{E}}$ and $\mathrm{V}_{2}$ during hypoxia have been previously described and can explain the observed results $(31,32)$. The experimental conditions were also different. In our previous study, the piglets were lightly restrained while in the present study, the rats were unrestrained inside the plethysmograph allowing the use of behavioral thermoregulation to compensate for changes in body temperature and metabolism, a physiologic response well developed in rats (33).

A similar decrease in $\mathrm{V}_{\mathrm{E}}$ was observed at 30 min of hypoxia after endotoxin administration and this was accompanied by a decrease in $\mathrm{VO}_{2}$ in all study groups. However, the fall in $\mathrm{Vo}_{2}$ at $30 \mathrm{~min}$ of hypoxia after endotoxin administration was significantly greater in the L-NAME and 7-NI groups suggesting that, after NOS blockade, the animals were able to maintain some degree of hyperventilation in relation to their metabolism. This response could be related to the larger decrease in Tb observed in the L-NAME and 7-NI treated animals. A similar effect was described in rats exposed to hypoxia at low $\mathrm{Tb}$, showing a greater hypometabolic response when compared with the change in $\mathrm{V}_{\mathrm{E}}$ (34). The decreases in $\mathrm{VO}_{2}$ and $\mathrm{Tb}$ observed after NOS blockade in this study are also supported by previous studies in rats that have shown that NO mediates thermogenesis in mammals $(35,36)$.

Additional mechanisms possibly contributing to the attenuation in the VRH during endotoxemia include lung injury and alterations on the contractile function of the respiratory muscles. Endotoxemia-induced lung injury is manifested by impaired gas exchange and changes in lung mechanics. We did not measure lung mechanics in this experiment. However, in a piglet model using low-dose E. coli endotoxin, we noted similar changes in compliance and resistance with hypoxia before and after endotoxemia, making this possibility unlikely as a mechanism for the attenuated VRH (3). Although endo- toxin can induce diaphragmatic dysfunction, the dose necessary to produce such changes is very high $(15 \mathrm{mg} / \mathrm{kg})(37)$, when compared with the low dose used in our study (25 $\mu \mathrm{g} / \mathrm{kg}$ ). Consequently, it is unlikely that changes in the contractile function of the respiratory muscles could explain the depression in the VRH observed in the present study.

There are some limitations with the use of whole body plethysmography in unrestrained animals. Measurements of changes in $V_{T}$ can be difficult, due to the fact that $V_{T}$ estimation is dependent on factors such as chamber integrity, choice of bias flow, chamber size, and ambient temperature. In the present study, the animals were studied under similar daily environmental circumstances (i.e. chamber size, ambient temperature, source and type of gas, method of administration, being stable and equal). Since $\mathrm{V}_{\mathrm{T}}$ measurements are qualitative and not absolute values, the results are presented as percent change from baseline. This would make a constant error factor unimportant, as previously suggested by other investigators $(38,39)$. Despite these technical limitations, we believe that our results are valid because the VRH before endotoxin was similar in the three study groups, suggesting that the observed differences in VRH after endotoxin reflect the biologic changes induced by L-NAME or 7-NI.

In conclusion, these data suggest that the attenuation in the VRH induced by $E$. coli endotoxin administration in young rats is in part mediated by an inhibitory effect of NO on the respiratory control mechanisms. This effect is modulated through activation of the endothelial NOS, in as much as L-NAME reversed the ventilatory depression whereas nNOS inhibition had no effect on the response.

\section{REFERENCES}

1. Fanaroff AA, Korones SB, Wright LL, Verter J, Poland RL, Bauer CR, Tyson JE, Philips JB 3rd, Edwards W, Lucey JF, Catz CS, Shankaran S, Oh W 1998 Incidence, presenting features, risk factors and significance of late onset septicemia in very low birth weight infants. The National Institute of Child Health and Human Development Neonatal Research Network. Pediatr Infect Dis J 17:593-598

2. Preas HL 2nd, Jubran A, Vandivier RW, Reda D, Godin PJ, Banks SM, Tobin MJ, Suffredini AF 2001 Effect of endotoxin on ventilation and breath variability. Role of cyclooxygenase pathway. Am J Respir Crit Care Med 164:620-626

3. McDeigan GE, Ladino J, Hehre D, Devia C, Bancalari E, Suguihara C 2003 The effect of Escherichia coli endotoxin infusion on the ventilatory response to hypoxia in unanesthetized newborn piglets. Pediatr Res 53:950-955

4. Prandota J 2004 Possible pathomechanisms of sudden infant death syndrome: key role of chronic hypoxia, infection/inflammation states, cytokine irregularities, and metabolic trauma in genetically predisposed infants. Am J Ther 11:517-546

5. Ali A, Goldberg RN, Suguihara C, Huang J, Martinez O, Feuer W, Bancalari E 1996 Effects of ATP-magnesium chloride on the cardiopulmonary manifestations of group B streptococcal sepsis in the piglet. Pediatr Res 39:609-615

6. Hussain SN 1998 Respiratory muscle dysfunction in sepsis. Mol Cell Biochem 179:125-134

7. McCuskey RS, Urbaschek R, Urbaschek B 1996 The microcirculation during endotoxemia. Cardiovasc Res 32:752-763

8. Vincent JL, Zhang H, Szabo C, Preiser JC 2000 Effects of nitric oxide in septic shock. Am J Respir Crit Care Med 161:1781-1785

9. Wolkow PP 1998 Involvement and dual effects of nitric oxide in septic shock 1998 Inflamm Res 47:152-166

10. Alderton WK, Cooper CE, Knowels RG 2001 Nitric oxide synthases: structure, function and inhibition. Biochem J 357:593-615

11. Salvemini D, Korbut R, Anggard E, Vane J 1990 Immediate release of a nitric oxide-like factor from bovine aortic endothelial cells by Escherichia coli lipopolysaccharide. Proc Natl Acad Sci U S A 87:2593-2597

12. Iwase K, Miyanaka K, Shimizu A, Nagasaki A, Gotoh T, Mori M, Takiguchi M 2000 Induction of endothelial nitric-oxide synthase in rat brain astrocytes by systemic lipopolysaccharide treatment. J Biol Chem 275:11929-11933

13. Reid G 2000 Association of sudden infant death syndrome with grossly deranged iron metabolism and nitric oxide overload. Med Hypotheses 54:137-139

14. Blood-Siegfried J, Nyska A, Geisenhoffer K, Lieder H, Moomaw C, Cobb K, Shelton B, Coombs W, Germolec D 2004 Alteration in regulation of inflammatory response to influenza a virus and endotoxin in suckling rat pups: a potential 
relationship to sudden infant death syndrome. FEMS Immunol Med Microbiol 42:85-93

15. Gozal D, Gozal E, Gozal YM, Torres JE 1996 Nitric oxide synthase isoforms and peripheral chemoreceptor stimulation in conscious rats. Neuroreport 7:1145-1148

16. Iturriaga R, Villanueva S, Mosqueira M 2000 Dual effects of nitric oxide on cat carotid body chemoreception. J Appl Physiol 89:1005-1012

17. Prabhakar NR 1999 NO and CO as second messengers in oxygen sensing in the carotid body. Respir Physiol 115:161-168

18. Dreshaj IA, Haxhiu MA, Martin RJ, Young JK 2003 The basomedial hypothalamus modulates the ventilatory response to hypoxia in neonatal rats. Pediatr Res 53:945949

19. Pappenheimer JR 1977 Sleep and respiration of rats during hypoxia. J Physiol 266:191-207

20. Cohen G, Gressens P, Gallego J, Gauthier C 2002 Depression of hypoxic arousal response in adolescent mice following antenatal vasoactive intestinal polypeptide blockade. J Physiol 540:691-699

21. Hamrahi H, Stephenson R, Mahamed S, Liao KS, Horner RL 2001 Regulation of sleep-wake states in response to intermittent hypoxic stimuli applied only in sleep. J Appl Physiol 90:2490-2501

22. Gautier H, Bonora M 1994 Ventilatory and metabolic responses to cold and CO-induced hypoxia in awake rats. Respir Physiol 97:79-91

23. Romanovsky AA, Shido O, Sakurada S, Sugimoto N, Nagasaka T 1996 Endotoxin shock: thermoregulatory mechanisms. Am J Physiol 270:R693-R703

24. Traystman RJ, Moore LE, Helfaer MA, Davis S, Banasiak K, Williams M, Hurn PD 1995 Nitro-L-arginine analogues: dose- and time-related nitric oxide synthase inhibition in brain. Stroke 26:864-869

25. Gozal D, Gozal E 1999 Episodic hypoxia enhances late hypoxic ventilation in developing rat: putative role of neuronal NO synthase. Am J Physiol 276:R17-R22

26. Iturriaga R, Alcayaga J 2004 Neurotransmission in the carotid body: transmitters and modulators between glomus cells and petrosal ganglion nerve terminals. Brain Res Rev 47:46-53
27. Haxhiu MA, Chang CH, Dreshaj IA, Erokwu B, Prabhakar NR, Cherniack NS 1995 Nitric oxide and ventilatory response to hypoxia. Respir Physiol 101:257-266

28. Gozal D, Gaultier C 2001 Evolving concepts of the maturation of central pathways underlying the hypoxic ventilatory response. Am J Respir Crit Care Med 164:325329

29. Olsson A, Kayhan G, Lagercrantz H, Herlenius E 2003 IL-1 $\beta$ depresses respiration and anoxic survival via a prostaglandin-dependent pathway in neonatal rats. Pediatr Res 54:326-331

30. Albelda SM, Elias JA, Levine EM, Kern JA 1989 Endotoxin stimulates plateletderived growth factor production from cultured human pulmonary endothelial cells Am J Physiol 257:L65-L70

31. Mortola JP, Rezzonico R, Lanthier C 1989 Ventilation and oxygen consumption during acute hypoxia in newborn mammals: a comparative analysis. Respir Physiol 78:31-43

32. Mortola JP, Matsuoka T, Saiki C, Naso L 1994 Metabolism and ventilation in hypoxic rats: effect of body mass. Respir Physiol 97:225-234

33. Gordon CJ 1990 Thermal biology of the laboratory rat. Physiol Behav 47:963-991

34. Hinrichsen CF, Maskrey M, Mortola JP 1998 Ventilatory and metabolic response to cold and hypoxia in conscious rats with discrete hypothalamic lesions. Respir Physiol 111:247-256

35. Malik SS, Fewell JE 2003 Thermoregulation in rats during early postnatal maturation: importance of nitric oxide. Am J Physiol Regul Integr Comp Physiol 285:R1366-R1372

36. Kamerman PR, Laburn HP, Mitchell D 2003 Inhibitors of nitric oxide synthesis block cold-induced thermogenesis in rats. Can J Physiol Pharmacol 81:834-838

37. Anderson JL, Williams G, Head SI 2001 The role of nitric oxide in diaphragmatic dysfunction in endotoxemic rats. Muscle Nerve 24:30-36

38. Mortola JP, Frappell PB 1998 On the barometric method for measurements of ventilation, and its use in small animals. Can J Physiol Pharmacol 76:937-944

39. Jacky JP 1980 Barometric measurement of tidal volume: effects of pattern and nasal temperature. J Appl Physiol 49:319-325 